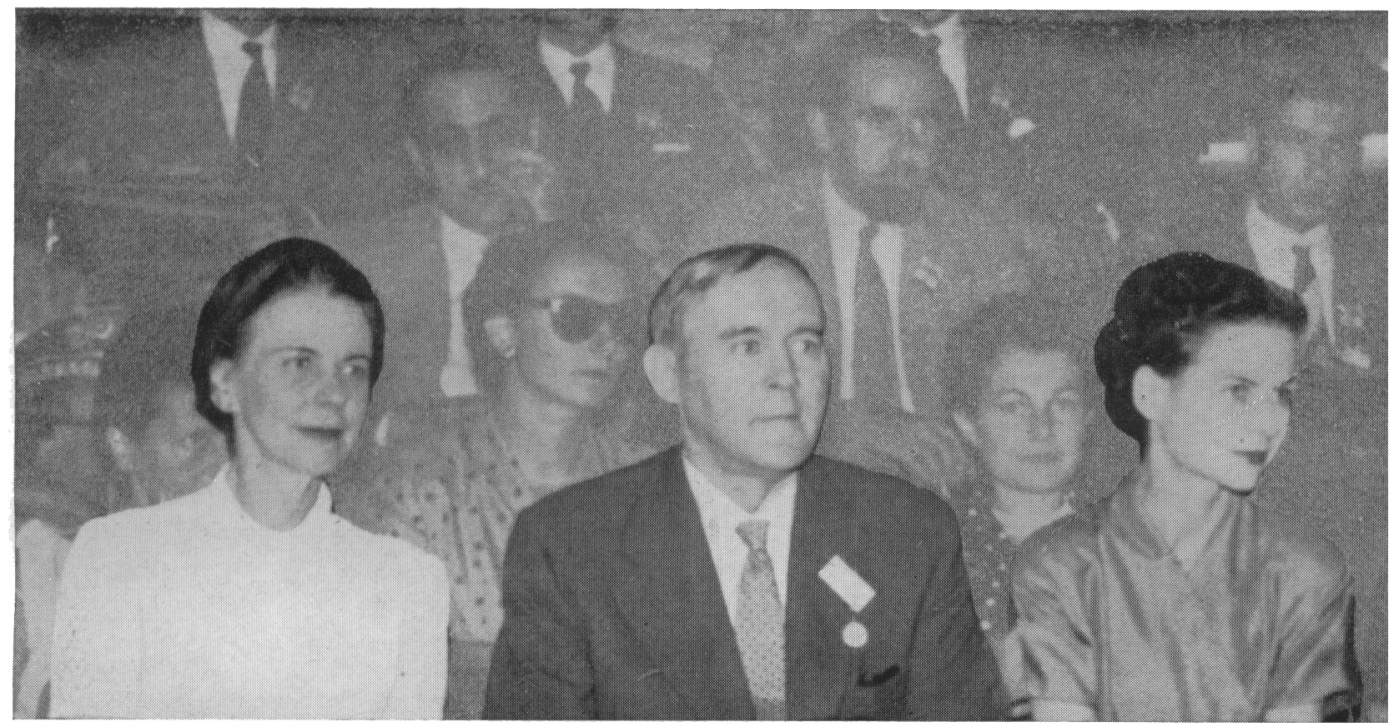

\title{
Gunnar Dahlberg
}

(in memoriam)

Mentre in Copenhagen stava per aprirsi il First Congress of Human Genetics, il 25 luglio 1956, moriva in Uppsala il Prof. Gunnar Dahlberg. Ma la notizia della sua morte è stata per lo più conosciuta solo dopo qualche tempo e perciò l'ombra di questa morte, che tale sarebbe stata, non scese sul Congresso, al quale pochi più di Dahlberg avrebbero avuto ragione di partecipare e di primeggiarvi.

La figura di Dahlberg si inscrive nella storia della Genetica come tipico esponente della Genetica Umana nel periodo in cui questa, nata dalla Genetica Naturalistica, si apprestava a qualificarsi, ad evolversi ed a generare la Genetica Medica. Non è senza significato il fatto che Dahlberg, succeduto a Lundborg nella direzione dell'Istituto di Genetica di Uppsala sia stato il Presidente e l'invisibile Organizzatore del Congresso di Genetica che si tenne in Stoccolma nel i948. Come Presidente impersonava la funzione di uno scienziato che, pur essendo genetista umano, conosceva profondamente $i$ settori naturalistici della Genetica e ne disponeva lo sviluppo attraverso le sezioni di competenza dell'ottavo Congresso. Come Organizzatore purtroppo era invisibile perchè fin da allora il male lo attanagliava ed egli agiva per le interposte persone dei suoi Allievi e della sua ammirabile Consorte.

$\mathrm{Ma}$ anche se la morsa del male non abbandonò la sua presa e attraverso quasi 
dieci anni lo condusse immaturamente alla tomba, il suo spirito rimase vigilante ed attivo fino all'estremo portando un contributo tanto più significativo, quanto più maturato nel sacrificio e nel superamento di sè.

Così è che il sorgere della Scuola Romana di Genetica Medica fu salutato dal Dahlberg con dichiarato favore e noi lo ricordiamo qui fra gli eminenti Scienziati che diedero il nome e la collaborazione alla nostra "Acta Genetica Medicae et Gemellologicae " fin dal suo sorgere.

Nel ${ }^{\circ}$ numero del I Anno di A. Ge. Me. Ge., Egli volle essere presente con un lavoro in lingua tedesca: «Die Tendenz zu Zwillingsgeburten », contributo che desideriamo rammentare non solo per il suo significato di apprezzamento e di solidarietà scientifica, ma altresì per il suo argomento rivolto agli studi del fenomeno gemellare nella specie umana, di cui Egli fu un antesignano. La monografia pubblicata da Gunnar Dahlberg nel 1926 "Twin births and twins from a hereditary point of tview », rimane una tappa fondamentale nel cammino della gemellologia.

Non fu quindi con sorpresa, ma certo non senza ammirazione, che abbiamo salutato Dahlberg nel I953 a Roma in occasione dell'inaugurazione dell'Istituto Mendel. Egli si era recato al nono Congresso di Genetica in Bellagio gravemente impedito nella parola e nei movimenti, ma ciononostante aveva voluto venire a Roma per prendere un contatto personale con l'ambiente romano rivolto agli studi della genetica medica e dalla gemellologia.

Benchè ammalato, venne a casa nostra e successivamente nell'Istituto Mendel dove partecipò alla seduta inaugurale; è precisamente questa sua presenza nell'Aula Magna del nostro Istituto che vogliamo rievocare e consegnare alla Rivista con la fotografia che pubblichiamo nella quale, a lato del Dahlberg, sono la Signora e la Figlia. L'estrema attività di Gunnar Dahlberg fu rivolta a fondare e ad avviare una nuova Rivista Internazionale di Genetica presso l'editore svizzero Karger. Si tratta della Rivista "Acta Genetica et Statistica Medica » che è ormai al $6^{\circ}$ volume e che attualmente è stata confidata alla dotta e qualificata direzione del Prof. Tage Kemp.

Con animo commosso noi segnamo la croce della morte accanto al nome di Dahlberg, ma vogliamo che il suo nome rimanga nel Comitato di Redazione come rimane in noi la sua memoria di uomo retto e coraggioso, amante della scienza ed aperto verso ogni nuova corrente indirizzata alla scoperta del Vero.

\section{GedDA}

